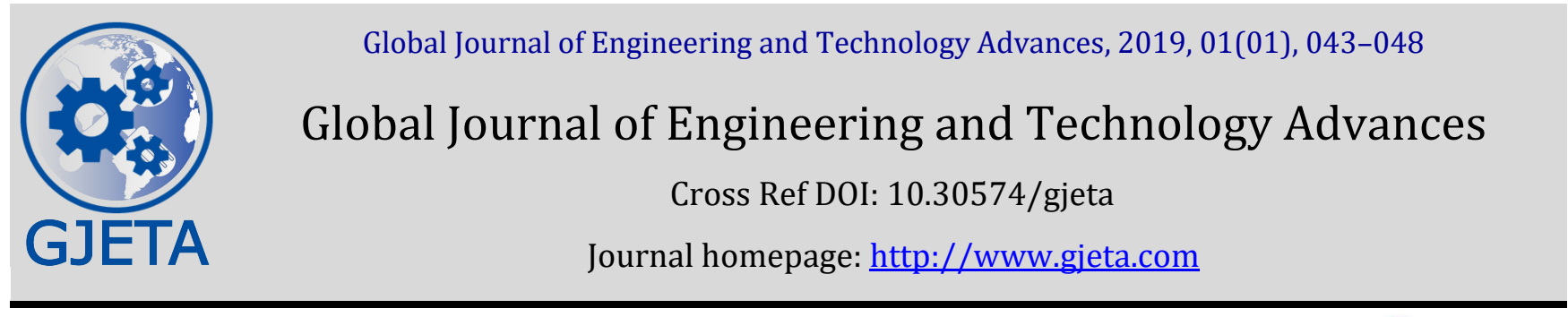

(RESEARCH ARTICLE)

\title{
Development of the algorithm of calculation of reactive power by harmonic components
}

Kholiddinov Ilkhombek Khosiljonovich ${ }^{1,}{ }^{*}$, Shaismatov Sayfulla Ergashevich ${ }^{2}$ and Eraliev Abdunabi Khakimovich ${ }^{1}$

${ }^{1}$ Department of Power Engineering Faculty of Energy Ferghana Polytechnic Institute, Uzbekistan.

${ }^{2}$ Department of Electrical Networks and Systems Tashkent State Technical University, Uzbekistan.

Publication history: Received on 21 October 2019; revised on 13 December 2019; accepted on 23 December 2019

Article DOI: https://doi.org/10.30574/gjeta.2019.1.1.0006

\begin{abstract}
The article presents the development of the algorithm for the study of higher harmonics of current and voltage. Research includes the harmonic interaction between transducers and power systems, as well as the simulation of higher harmonics research. The information on the application of the algorithm on the modern tool base is given.
\end{abstract}

Keywords: Electrical network; Electrical equipment; Power quality; Voltage and current asymmetry; Measuring device.

\section{Introduction}

One of the main objectives of this work is the development of a method to account for losses caused by higher harmonic currents and voltages. At the same time, it is obvious that these components of losses must be assessed individually, due to their different weight significance, a significant difference in terms of distribution, on the equivalent circuits applied to them and their parameters.

There is no doubt that a special need to account for such losses arises when the requirements of G0ST 32144-2014 are violated, which set different standards not only for each harmonic, but also depending on the nominal voltage of the network.

It is from these positions that it is impossible not to evaluate the existing methods for calculating capacities, their applicability to balance equations, and the calculation of losses caused by the distortions under consideration.

The existing methods for determining the power components, depending on the mathematical apparatus used can be divided into: spectral, integral, exchange power method, equivalent sinusoid method, main harmonic method, energyflow theories, etc.

\section{Methods}

In spectral methods, the power components are determined through the harmonic components of the currents and voltages.

The active power of a periodic current of arbitrary shape is defined as the average power for a period [1,2]:

\footnotetext{
${ }^{*}$ Corresponding author

E-mail address: holiddinov_ilhombek@mail.ru
} 


$$
P=\frac{1}{T} \int_{0}^{T} U I d t
$$

Indeed, if the instantaneous values of voltage and current are expressed in the form of trigonometric series, then we obtain $[1,2]$ :

$$
P=\frac{1}{T} \int_{0}^{T}\left[\sum_{n=0}^{\infty} U_{n} \sin (n \omega t)\right]\left[\sum_{n=0}^{\infty} I_{n} \sin \left(n \omega t+\varphi_{n}\right)\right] d t
$$

The average for the period value of the product of the instantaneous values of sinusoids of various frequencies is zero, and after integration $[1,2]$ :

$$
P=\sum_{n=0}^{\infty} U_{n} I_{n} \cos \varphi_{n}=\sum_{n=0}^{\infty} P_{n}
$$

From this expression it follows an important conclusion that the average power of a non-sinusoidal current is equal to the sum of the average powers of the individual harmonics. Thus, expression (3) fully meets the stated requirements, allowing to calculate active power losses both individually for each harmonic and integrally. The active energy, developed or consumed, is determined in a similar way during T [1, 2]:

$$
W_{a}=T \sum_{n=0}^{\infty} U_{n} I_{n} \cos \varphi_{n}=T \sum_{n=0}^{\infty} P_{n}
$$

In addition to the active power determined by the formula 4, in accordance with the theories of K. Budenau and S. Frieze $[3,4]$ in the mode with non-sinusoidal currents and voltages will also be present reactive power $Q$ and power distortion $\mathrm{T}$, determined by the formulas $[2,4]$ :

$$
\begin{aligned}
Q & =\sum_{n=1}^{n} Q_{n}=\sum_{n=1}^{n} U_{n} I_{n} \sin \varphi_{n} \\
T & =\sqrt{\sum_{n \neq m} U_{n}^{2} I_{m}^{2}+U_{m}^{2} I_{n}^{2}-2 U_{n} U_{m} I_{n} I_{m} \cos \left(\varphi_{n}-\varphi_{m}\right)} .
\end{aligned}
$$

Here, the calculated expression (5) allows us to estimate the reactive power for individual harmonics. However, on the network element for which the reactive power is calculated, there arises another, besides the active component, the power component - the distortion power. This value has no physical meaning and cannot be measured. Therefore, the expression (5) cannot be considered reliable [2].

N.Casters and V.Mur [3], analyzing the possibility of reactive power compensation in linear and nonlinear circuits in any energy process, determined the inductive and capacitive reactive powers: $Q_{L}=\frac{U(\bar{u}(t) i(t))}{\bar{U}}, Q_{C}=\frac{U(\dot{u}(t) i(t))}{\dot{U}}$, where U is the effective value of voltage; $\bar{u}(t)$ and $\bar{U}$ are the instantaneous and square root values of the variable $\int u d t ; \dot{u}(t)$ и $\dot{U}$; and is the instantaneous and square root value $\frac{d u}{d t}[2,5]$.

Reactive powers according to Custer and Moore in the frequency domain are equal to:

$$
Q_{L}=\sum_{n=1}^{n} \frac{\omega_{L}}{\omega_{n}} U_{n} I_{n} \sin \varphi_{n}, \quad Q_{C}=\sum_{n=1}^{n} \frac{\omega_{n}}{\omega_{C}} U_{n} I_{n} \sin \varphi_{n},
$$

where two fixed frequencies are entered $\omega_{L}=\frac{U}{\bar{U}}$ and $\omega_{\mathrm{C}}=\frac{\dot{U}}{U}$ We get: 


$$
Q_{L}=\sum_{n=1}^{n} \frac{1}{n} Q_{n} \text { и } Q_{C}=\sum_{n=1}^{n} n Q_{n} .
$$

Inductive and capacitive reactive components can be compensated by parallel inclusion of the corresponding element. Residual reactive components cannot be compensated in this way $[2,5]$.

In the integral methods of M. Iliovisi [3] and O.A. Mayevsky proposed expressions for calculating the integral components of the total power. Reactive power at non-sinusoidal values is equal to:

$$
Q= \pm \frac{1}{2 \pi} \oint i(u) d u ; \quad Q= \pm \frac{1}{2 \pi} \oint u(i) d i
$$

When bringing the integral value of reactive power to a certain specific frequency [3]:

$$
Q=\sum_{n=1}^{n} \frac{\omega_{n}}{\omega_{\Pi}} Q_{n},
$$

where $\omega_{n_{-}}$is the harmonic frequency, $\omega_{\Pi}$ is the frequency of reduction. Then for the $\mathrm{n}$-th order harmonic:

$$
Q=\sum_{n=1}^{n} n Q_{n} .
$$

From these expressions it follows, that the reactive powers of the individual harmonics are not equivalent, and the reactive powers of the individual harmonics are included in the expressions of the total reactive power with a coefficient proportional to their frequency [2].

The harmonic interpretation of reactive power, used for the first time by Mayevsky, consists in the proportionality of the area of the current-voltage characteristic of a two-pole device described over the period, of reactive power; $Q=$ $-\frac{1}{2 \pi} \oint i d u= \pm \frac{1}{2 \pi}|s| m_{u} m_{i}$, where $\mathrm{s}$ - is the area of the current-voltage characteristic, $\mathrm{m}_{\mathrm{u}}$ и $\mathrm{m}_{\mathrm{i}}-$ are the scales along the coordinate axes.

For the integral component of power, the law of energy conservation throughout the entire closed electric circuit is valid. K.S. Demirchyan also believes that the concept of reactive power should be linked to the intensity of the physical processes of the exchange of electromagnetic energy between the individual elements of the electrical circuit $[2,3]$.

In the theory of E. Pille [3], the reactive power at a non-sinusoidal voltage is $Q=\frac{2 \pi}{T} \int_{0}^{T}(G-\mathrm{E}) d t$, where $\mathrm{G}$ - is the magnetic field energy, $\mathrm{E}$ is the electric field energy. With regard to the RL - chain $Q=\omega L I^{2}$, for RC - chain $Q=-\omega C U^{2}$ , and for RLC sequences - chain: $Q=\sum_{n=1}^{n} \frac{1}{n} U_{n} I_{n} \sin \varphi_{n}$ [2].

E. Pille considers reactive power as a component of total power along with active power and distortion power. He believes that the power of distortion has no physical meaning, neither calculation nor measurement is supplied and cannot be compensated.

F.P. Zharkov proposes to use the concept of "exchange capacity" [3]. When consumed, energy is converted and accumulated; when the stored energy is returned, partial conversion of energy into other forms takes place.

The value of $\frac{d W}{d t}=\frac{W_{\max }-W_{\min }}{T}$ F.P. Zharkov calls the value of the exchange energy averaged over the interval T. In $[2,5]$ it is noted that in this formulation F.P. Zharkov determined not the exchange $W_{\text {exch }}=W_{\max }-W_{\min }$, but the maximum accumulated energy. For an RL circuit with a sinusoidal voltage and period T, the reactive power is $Q=\frac{2 \pi}{T}\left(W_{\max }-\right.$ $\left.W_{\min }\right)$. With such a record, $Q=\omega\left(W_{\max }-W_{\min }\right)[2,5]$.

According to K.S. Demirchyan distribution of these formulas to non-sinusoidal modes is possible using the method of equivalent sinusoids. In his opinion, to assess the intensity of the exchange processes, it is advisable to use the exchange 
power $Q_{\text {exch }}=\frac{\text { Wexch }}{T}$, where Wexch - is the exchange energy for a period with sinusoidal processes, equal to twice the maximum value of the instantaneous accumulated electromagnetic energy.

I.V. Zhezhelenko and Yu.L. Saenko believes that reactive power in the system should be proportional to Joule losses [4]. They recommend determining reactive power based on the main harmonics of current and voltage. The use of the concept of reactive power only for sinusoidal modes is also suggested by V.A. Venikov, L.A. Zhukov, N.A. Melnikov and other scientists [3].

Analyzing the approaches designed by these authors, it should be noted that most of the methods come down to the possibility of calculating the reactive power for the harmonic components. The resulting imbalance is "written off" to the power, which has no physical meaning, and therefore can be attributed to the methodological error of the calculation. It will certainly affect the accuracy of the measurement tools, which will increase with increasing harmonic composition of voltage and current $[2,5]$.

\section{Results and discussion}

For a branched chain containing linear and nonlinear elements, the reactive power balance is described by the expression $[2,5]$ :

$$
U_{1} I_{1} \sin \varphi_{1}=\sum_{n=1}^{n} n Q_{(n)}+\sum_{n=1}^{n} n \Delta U_{S(n)} I_{(n)} \sin \varphi_{S(n)},
$$

Where $U_{1} I_{1} \sin \varphi_{1}$ - reactive power source; $\sum_{n=1}^{n} n Q_{(n)}$-reactive power nonlinear load; $\sum_{n=1}^{n} n \Delta U_{S(n)} I_{(n)} \sin \varphi_{S(n)}$ reactive power losses in network elements.

An algorithm has been developed for calculating the source's reactive power based on (11), which allows to take into account the reactive power of a non-linear load and the loss of reactive power in the network elements. This allows a more objective assessment of the reactive power of the source, which occurs when a number of electrical receivers operate, powered from the network with an asymmetric voltage system (Fig. 1) [5, 6].

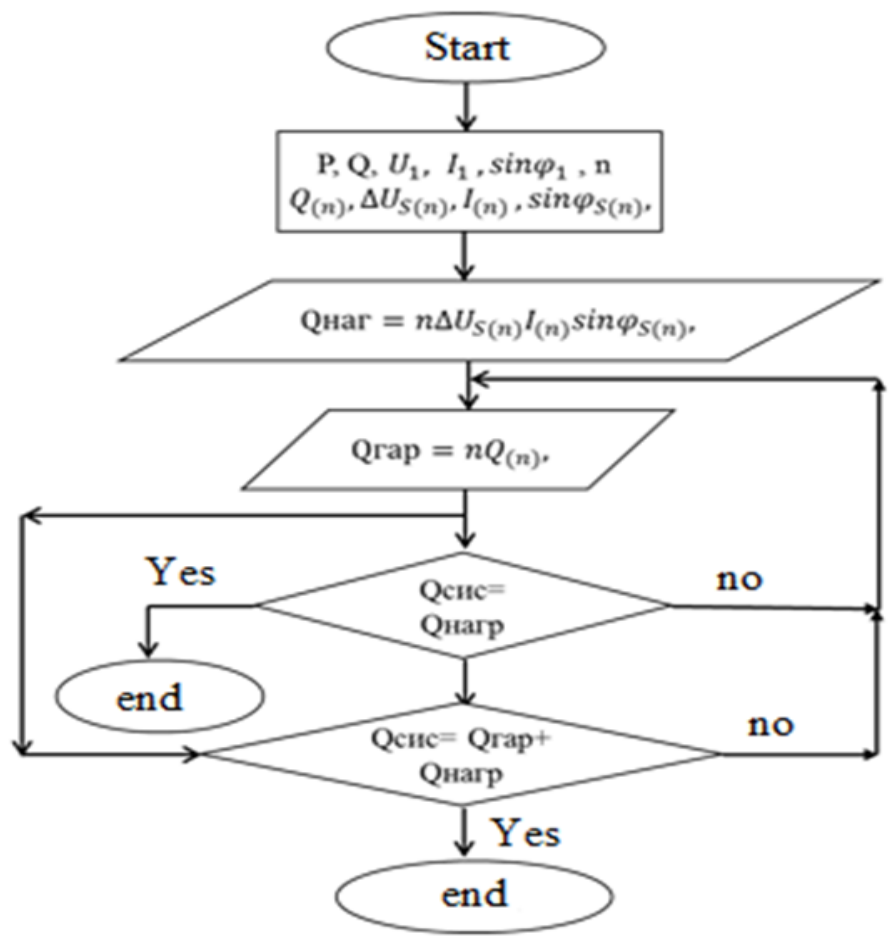

Figure 1 Structural scheme for calculating the balance of reactive power 
The presented structural diagram (Fig. 1) of the algorithm for calculating reactive power losses with regard to higher harmonics works as follows. The source data obtained determines the reactive power losses in the network elements and compares with the value of the reactive power from the source, if these values are equal, then the calculation ends. If the condition is not met, then the reactive power of the nonlinear load with the existing harmonics is determined and summed with the reactive power losses in the network elements, then these values are compared with the value of the reactive power from the source. If these values are equal, then the calculation ends. If the conditions are not met, then the reactive power of the nonlinear load with the existing harmonics is redetermined until the required balance conditions are fulfilled.

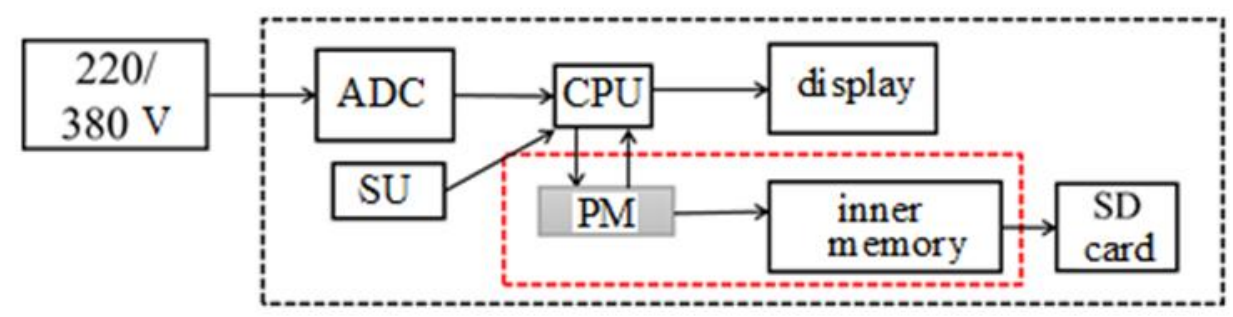

ADC - block input circuits, SU - control system (keyboard), CPU - central processor; PM-microprocessor performs calculation of reactive power losses taking into account higher harmonics.

Figure 2 Structure of reactive power measurement device "Malika-01"

The author modernized the multifunctional device "Malika-01", made on a modern microprocessor base to account for the higher harmonic components of the current and voltage in the electrical system. The algorithm for calculating the reactive power losses taking into account higher harmonics using the RM block, presented in Fig. 2 [5, 6], was laid.

\section{Conclusion}

Analysis of existing theories of power, as well as methods for describing electrical transformations in non-sinusoidal modes, allows us to draw the following conclusions $[5,8,9]$ :

- none of the existing methods for calculating the components of reactive power does not allow to fully describe the physical processes associated with energy transformations in non-sinusoidal modes;

- the most appropriate approach should be considered, in which the power components are determined by the corresponding currents and voltages of higher harmonics:

for four and five wire networks:

$$
P_{n \Sigma}=\sum_{A, B, C} \sum_{n=2} U_{n} I_{n} \cos \varphi_{n}, \quad Q_{n \Sigma}=\sum_{A, B, C} \sum_{n=2} n U_{n} I_{n} \sin \varphi_{n}
$$

for three-wire networks:

$$
P_{n \Sigma}=\sum_{n=2} \sqrt{3} U_{n} I_{n} \cos \varphi_{n}, \quad Q_{n \Sigma}=\sum_{n=2} \sqrt{3} n U_{n} I_{n} \sin \varphi_{n}
$$

-to estimate additional losses, it is necessary to investigate by measuring in existing electrical networks of various voltages in the presence of significant distortions of the sinusoidality of currents and voltages.

\section{Compliance with ethical standards}

\section{Disclosure of conflict of interest}

The authors declare that they have no conflict of interest. 


\section{References}

[1] Bessonov LA. Theoretical Foundations of Electrical Engineering. (2014). Electrical circuits / L. A. Bessonov. $12^{\text {th }}$ ed., Rev. and add. - M.: Yurayt, 701.

[2] R Shamanov. (2003). Development of a methodology for assessing the impact of power quality on power losses and energy in electrical networks: dis. Ph.D. - Moscow, 155.

[3] Bakov Yu V. (1999). AC power alternating current. - Ivanovo State Energy university, 200.

[4] Zhezhelenko IV. (2007). Selected Issues of Non-sinusoidal Regimes in Electric Networks of Enterprises: Monograph / Ed. I.V. Zhezhelenko. - M.: Energyatom publish, 296.

[5] Kholiddinov IKh. (2017). Methods and algorithms to ensure the quality of electricity in asymmetric modes in low-voltage electrical networks: dis. Ph D Tech. - Tashkent. 119.

[6] Patent UZ FAP № 01166. A device for recording additional electric power losses during unbalance of loads in low-voltage electrical networks. / Allaev K.R., Shaismatov S.E., Kholiddinov I. Kh. // 05/22/2015.

[7] Zhezhelenko IV. (2010). Electrical losses from higher harmonics in power supply systems // Elektrika. - № 4.36.

[8] Kholiddinov I. Kh. Electric power quality analysis 6-10/0.4 kV distribution networks. Journal Energy and Power Engineering (EPE) 2016 y. USA

[9] Kholiddinov I.Kh., Ponomarenko O.I. Provision of the instrument base of the power quality control system in modern power supply systems // Universum: Engineering: electron. Scientific journal 2016. -№8 (29). -FROM. 66-70. 7universum.com/en/tech/archive/item/3527

\section{How to cite this article}

Kholiddinov IK, Shaismatov SE and Eraliev AK. (2019). Development of the algorithm of calculation of reactive power by harmonic components. Global Journal of Engineering and Technology Advances, 1(1), 43-48. 